\title{
GIANT RETINAL TEAR MANAGEMENT AT REFFERAL EYE HOSPITAL
}

\author{
Mia Purnama, Iwan Sovani, Arief S Kartasasmita, Erwin Iskandar, Rova Virgana, Grimaldi Ihsan \\ Universitas Padjadjaran/Cicendo National Eye Hospital
}

\begin{abstract}
Introduction: to report the characteristics, management and outcome in giant retinal tear (GRT) associated retinal detachment patients at Cicendo Eye Hospital

Methods: this retrospective study was performed on medical records who had undergone retinal detachment surgery between January 2014 and March 2017. Age, sex, etiologies, size of GRT, quadrant involvement, lens status, proliferative vitreo-retinopathy (PVR), managements and outcomes were evaluated in association with giant retinal tears
\end{abstract}

Result: Twenty-six patients (23 males, 3 females) age between 11-59 years with follow up from 2 months to 18 months were enrolled in this study. Twenty-five eyes have retinal detachment with macular involvement and 11 patients had high myopia. Majority of patients had $90^{\circ}$ of GRTs. Most retinal tears were located at temporal quadrant (73\%). Nineteen patients had undergone pars plana vitrectomy (PPV) and 7 patients had combined PPV with encircling buckle. Fifteen patients had used heavy liquid, 24 patients had silicon oil and 2 had gas tamponade. Intraoperative complications included lens trauma, retinal slippage and choroidal detached were found in 1 eye respectively. Fourteen eyes had recurrent retinal detachment. At the last follow up, 14 patients had anatomically attached retina. Twelve patients had total retinal detachment and marked PVR. Five fellow eyes were treated with prophylactic laser. Visual acuity improved in 11 eyes.

Conclusion: Giant retinal tears were more common in patients with high myopia. Management of GRT currently with PPV and PPV combined with encircling buckle. The success rate of anatomy and visual acuity was less than other previous studies

Keywords: giant retinal tear, proliverative vitreo-retinopathy, pars plana vitrectomy

Cite This Article: PURNAMA, Mia et al. Giant Retinal Tear Management at Referral Eye Hospital. International Journal of Retina, [S.I.], v. 1, n. 1, sep. 2017. ISSN 2614-8536. Available at: https://www.ijretina.com/index.php/ijretina/article/view/5

*Correspondence to:

Arief F Kartasasmita,

Universitas Padjadjaran,

Cicendo National

Eye Hospital

a.kartasasmita@unpad.ac.id

\section{INTRODUCTION}

Giant retinal tear (GRT) is a full-thickness retinal break extending circumferentially for 90 degrees or greater of the retina associated with vitreous detachment. Their management poses significant challenges due to the many complications and technical difficulties involved for unfolding and sealing the retinal tear. ${ }^{1-4}$ Although GRT can occur spontaneously, they are often associated with a number of conditions; this include ocular trauma, high myopia, pseudophakia, hereditary conditions, for example, Marfan syndrome, Stickler syndrome, extensive Lattice degeneration and young age..$^{5,6}$ With such a large tear, the anterior insertion of the retina no longer offers peripheral support and the retina folds back on itself. The retina also tends to roll due to the absence of vitreous attachment. $^{7}$ 
A wide range of techniques have been used with varying degrees of success. Recently, common techniques used to treat GRT include primary vitrectomy with expansile gas or silicon oil tamponade, and combined scleral buckle-vitrectomies. The use of heavy liquid as an adjunctive tool has made unfolding and stabilization of the retina easier. The use of silicon oil has also been proven to be more effective in early visual rehabilitation and is also safer to exchange with perfluorocarbons. ${ }^{7}$

Proliverative vitreo-retinopathy (PVR) is one of the late complications of GRT, and the leading cause of surgical failure. Increased access to the exposed retinal pigment epithelium (RPE) allows greater spillage of cells and pigment into the vitreous cavity and on the retinal surface, thereby increasing the risk of PVR. ${ }^{8}$ Giant retinal tears in pediatric patients (younger than 16 years) are more likely to be associated with PVR which may be due to a delay in diagnosis or the greater wound healing response in children. ${ }^{8}$ Other mechanism predisposing to PVR include the presence of vitreous hemorrhage and breakdown of the blood-ocular barrier leading to production of cytokines which could further stimulate cellular proliferation. 8,9

Since GRT can adversely affect the prognosis of retinal detachment surgery, we decided to report patients who were operated for retinal detachment with GRT at our center and evaluate etiology, management and outcomes.

\section{PATIENTS and METHODS}

This is a retrospective study of 26 eyes of 26 patients between the ages of 11 and 59 years. These patients underwent surgery to repair rhegmatogenous retinal detachment due to GRT between January 2014 and March 2017 at the Cicendo Eye Hospital. Patients were included and all patients had more than 2 months of follow up. Giant retinal tear was defined as a retinal tear of $90^{\circ}$ or greater confirmed intraoperatively or by clinical examination. Of the excluded eyes, 2 had initial vitreous surgery elsewhere, 1 had undergone previous pars plana vitrectomy (PPV), and 1 had less than 1 month of follow up. All case notes were then reviewed.

Age, sex, address, etiology, lens status, size of GRT, quadrant involvement, macular involvement, PVR, and surgical procedure, usage of heavy liquid, tamponade, complications, length of follow up and prophylactic laser of fellow eye.

Outcome variables included visual acuity, rates of retinal re-attachment and incidence of PVR. The operating surgeon selected the surgical approach for the individual patient, and there was no defined protocol in this study. Complete anatomical success was defined as complete retinal attachment after silicone oil removal at the 2th or more postoperative month, while incomplete success was considered in eyes were the retina remained detached under silicon oil or redetached after silicon oil removal.

\section{RESULTS}

There were 26 eyes of 26 patients with rhegmatogenous retinal detachment associated with GRT. Of these, 23 were males and 3 females, with 14 right eyes and 12 left eyes being affected. Their age ranged between 11 and 59 years with a mean of 38.39 years (SD: 13.67). In all, 23 patients were coming from west java, 2 were from central java, and 1 from Aceh. Postoperative follow-up period ranged from 2 months to 18 months (mean 6,58 months $\pm 4,86$ ). The location of most retinal tears was temporal quadrant $(69 \%)$ of 18 eyes.

In all, 25 eyes (96\%) had a retinal detachment involving the macula with a significant drop in visual acuity. Altogether 11 eyes (42\%) had etiology of high myopia, 6 eyes (23\%) had history of blunt trauma, 7 eyes (27\%) of idiopathic, 1 eye (4\%) was Marfan's syndrome and 1 eye (4\%) was extensive Lattice degeneration.

A total of 14 eyes (54\%) affected right eye and 12 (46\%) left eye. Visual acuity of hand motion were 19 eyes (73\%), 5 eyes (19\%) were light perception, 1 eye (4\%) was 1 meter counting finger, 1 eye $(4 \%)$ was 0,4 . Lens status of phakic eye were 19 eyes (73\%), pseudophakic eye with posterior chamber intraocular lens were 7 eyes (27\%).

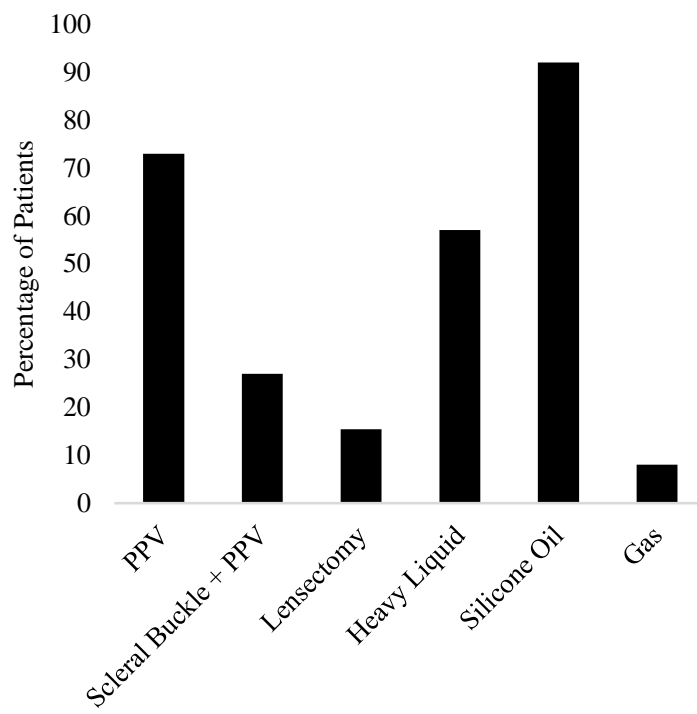

Figure 1. Initial Management for Patients Undergoing Surgery for Giant Retinal Tear-Associated Retinal Detachment

In all, 13 eyes (50\%) had GRT that extended $90^{\circ}$ circumferentially, with 11 eyes (42\%) had GRT that extended between $90^{\circ}$ and $180^{\circ}$ circumferentially and 2 eyes (8\%) had GRT greater than $180^{\circ}$.

At the first presentation, there were 17 eyes (65\%) with no PVR, 6 eyes (23\%) had PVR grade B, and 3 eyes $(11,5 \%)$ had PVR grade $C$. At the last surgeries found that incidence rate of PVR grade B was 23\% (6 eyes) and incidence rate of PVR grade C increased to $42 \%$ (11 eyes). 
A total of 19 eyes had undergone pars plana vitrectomy (PPV) and 7 eyes had undergone combined PPV with encircling scleral buckle. Attachment of retina after initial surgery of PPV were $42 \%$ (8/19 eyes) of cases and eyes after initial surgery of PPV with encircling buckle were $57 \%$ (4/7 eyes) of cases.

\section{TABLE 1. Baseline Characteristics of Patients Undergoing Surgery for Giant Retinal Tear- Related Retinal Detachments}

\begin{tabular}{ll}
\hline Baseline Parameter & $\mathrm{n}=26$ \\
\hline $\begin{array}{l}\text { Mean Age, Years (SD) } \\
\text { Age, Years (range) }\end{array}$ & $38.39(13.67)$ \\
& $11-59$ \\
\hline Gender, n (\%) & $23(88)$ \\
Male & $3(12)$ \\
Female & \\
& $6.58(4.86)$ \\
Mean Follow- Up, Months (SD) & $2-18$ \\
Length of follow up, Months (range) &
\end{tabular}

\begin{tabular}{ll}
\hline Etiologies, $\mathrm{n}(\%)$ & $11(42)$ \\
High Myopia & $7(27)$ \\
Idiopathic & \\
& \\
& $6(23)$ \\
Trauma & $1(4)$ \\
Extensive Lattice Degeneration & $1(4)$ \\
Marfan's Syndrome & \\
& \\
\hline Visual Acuity Pre-operative, n (\%) & $19(73)$ \\
Hand Motion & $5(19)$ \\
Light Perception & $1(4)$ \\
1 meter counting finger & $1(4)$ \\
0.4 &
\end{tabular}

\begin{tabular}{ll}
\hline Lens Status, n (\%) & $19(73)$ \\
Phakic & $7(27)$ \\
Pseudophakic & \\
& \\
\hline Quadrant Involvement, n (\%) & $18(69)$ \\
Temporal & $5(19)$ \\
Inferior & $2(8)$ \\
Superior & $1(4)$ \\
Nasal & \\
& \\
\hline Macular Involvement, n (\%) & $25(96)$ \\
Off & $1(4)$ \\
On & \\
& \\
\hline Proliferative vitreo-retinopathy, n (\%) & $17(65)$ \\
No Proliferative vitreo-retinopathy & $6(23)$ \\
B & $3(12)$ \\
C & \\
& \\
\hline Size of Giant Retinal Tear, n (\%) & $13(50)$ \\
$90^{\circ}$ & $11(42)$ \\
$90^{\circ}-180^{\circ}$ & $2(8)$ \\
$>180^{\circ}$ & \\
\end{tabular}

Retinal re-detachment after initial surgery of PPV were $58 \%$ (11/19 eyes) of cases and eyes after initial surgery of PPV with encircling buckle were $43 \%$ (3/7 eyes).
Lensectomy were performed on 4 eyes due to lens opacity, the rest were not done lensectomy. The use of heavy liquid in this study were obtained in 15 eyes and 11 eyes did not use heavy liquid.

Eyes with GRT in this study were given tamponade of silicon oil (SO) as much as 24 and 2 eyes were gases. The use of silicon oil in this study include the viscosity of 1000 centistoke given to 13 eyes, viscosity of 5000 centistoke given to 9 eyes and heavy SO given to 2 eyes. Gases tamponade that has been used in this study were SF6 and C3F8, both patient given this gasses tamponade experienced redetached retina and performed reoperation. Eyes with redetached retina after being given SO tamponade with viscosity of 1000 centistoke as much as 7 eyes, SO viscosity of 5000 centistokes were 5 eyes and heavy SO was 1 eye.

\section{TABLE 2. Intraoperative Data for Patients with Giant Retinal Tear-Related Retinal Detachments}

\begin{tabular}{ll}
\hline Intraoperative Parameter & Number $(\%)$ \\
\hline Tamponade & $1(4)$ \\
SF6 & $1(4)$ \\
C3F8 & $13(50)$ \\
Silicon Oil $1000 \mathrm{cs}$ & $9(34)$ \\
Silicon Oil 5000 cs & $2(8)$ \\
Heavy Silicon Oil & \\
& \\
Complication & $23(88)$ \\
No Complication & $1(4)$ \\
Lens Touch & $1(4)$ \\
Slippage Retina & $1(4)$ \\
Choroidal Detached & \\
& $21(81)$ \\
\hline Retinal condition at the end of initial surgery \\
Attached Retina Perfectly \\
Attached Retina Imperfectly & $5(19)$ \\
&
\end{tabular}

Various types of complications that had occurred intraoperative in this study include, lens touch in 1 eye, slippage in 1 eye with encircling buckle, and choroidal detached in 1 eye. Retinal condition at the end of surgery were obtained with 21 eyes were perfectly attached retina and 5 eyes were imperfectly attached retina due to the rest of subretinal fluid.

Visual acuity at the end of follow up varies from hand motion of 13 eyes, 1 until 6 meter counting finger were 10 eyes and more than 0.1 were 3 eyes. At the end of follow up period visual acuity improved in 11 (42\%) eyes, did not change in 14 (54\%) eyes and worsened in one (3.8\%) eye.

A total of 12 eyes (46\%) have no re-operation. Reoperation 1 time on 10 eyes (38\%) and re-operation 2 times on 4 eyes (15\%).

Last retinal condition at the end of follow up found 14 eyes (54\%) attached retina and 12 eyes (46\%) redetached 
retina. Prophylactic laser of fellow eye performed 5 eyes and the rest is not performed.

\section{DISCUSSION}

Giant retinal tear associated retinal detachment are relatively uncommon. These patients present a surgical challenge because of the high incidence of PVR and redetachment rate. Of the 1233 documented clinical encounters with retinal detachment underwent retinal reattachment surgery identified during the current study period, only $26(2 \%)$ patients were identified as having giant retinal tear.

TABLE 3

Anatomic and Visual Acuity Outcomes of Patients Undergoing Surgery for Giant Retinal tear-Related Retinal Detachments

\begin{tabular}{lc}
\hline $\begin{array}{l}\text { Outcomes Parameter } \\
(\%)\end{array}$ & Number \\
& \\
\hline Retinal attachment rates, n (\%) & $8(42)$ \\
Vitrectomy & $4(57)$ \\
Vitrectomy Combined Encircling Buckle & \\
\hline Recurrent Retinal Detachment & $14(54)$ \\
Re-operation 1 time & $10(38)$ \\
Re-operation 2 times & $4(16)$ \\
& \\
\hline Last Retinal Condition at Last Follow-Up & $14(54)$ \\
Attached Retina & $12(46)$ \\
Re-detached Retina & \\
& \\
\hline Final Visual Acuity & $13(50)$ \\
Hand Motion & $10(38)$ \\
1- 6 meter counting finger & $3(12)$ \\
$>0.1$ & \\
& \\
\hline Visual Acuity at Last Follow Up & $11(42)$ \\
Improved & $14(54)$ \\
Did not change & $1(4)$ \\
Worsened & \\
\hline
\end{tabular}

Giant retinal tears comprise about $1.5 \%$ of rhegmatogenous retinal detachments. The average age of incidence is 42 years. There is usually a higher incidence in males than females, with males comprising $72 \%$ of cases. About $54 \%$ of GRTs are idiopathic, $12.3 \%$ result from trauma, 25\% result from high myopia, and $14 \%$ result from other hereditary conditions such as Marfan's, Stickler-Wagner, and Ehrler Danlos syndromes. ${ }^{10}$

In the current study, a history of high myopia was the single most identifiable etiology in the study population (42\%). Marco et al., reported the GRT associated retinal detachment study with the greatest etiology of extensive lattice degeneration in 24 eyes (30\%) and high myopia were 21 eyes (27\%) of 79 cases. $^{5}$

Gonzalez and flyyn reported that trauma was a common associated factor in patients presenting with GRT associated retinal detachment. ${ }^{11}$
In addition to PPV, the use of encircling scleral buckle has been a point of controversy among vitreo-retinal surgeons. Scleral buckles are supposed to aid in reduction of traction within the vitreous base, thereby ensuring that the GRT do not extend or re-open. This reduces the chances of recurrence of retinal detachment due to the formation of fresh anterior retinal breaks or PVR. ${ }^{3,} 12$ However this procedure has also been implicated in complicating the closure of GRT due to distortion of the shape of the eye, fish-mouthing and tendency to increase 'slippage' of the retina posteriorly and the possible induction of PVR due to additional trauma. ${ }^{3,8}$ Conversely, other surgeons prefer adjunctive buckling in as a primary procedure aiming to reduce the failure rate. Their rationale was that scleral buckling reduces the early and late tractional forces and supports areas of un-detected retinal breaks. ${ }^{5}$ Meanwhile, other surgeonsreserve scleral buckling only for second intervention. ${ }^{3}$ In this study, we found that retinal re-attachment was successful with combine PPV with encircling buckle in 4/7 eyes (57\%) and PPV alone was $42 \%$ (8/19 eyes). Ambresin et al., reported the success rate of management of GRT with vitrectomy, internal tamponade, and peripheral 360 degrees photocoagulation of the retina without scleral buckle is high $(94.4 \%) .^{13}$

Proliferative vitreo-retinopathy has an incidence of up to $40-50 \%$ in association with GRT. This suggest that, unless these eyes have adequate management of PVR, there will be a high failure rate. ${ }^{1,2}$ An incidence of PVR at the first presentation in this study were $34 \%$ (9 eyes) and at the end of surgery were $65 \%$ (17 eyes). Dabour reported PPV combined with scleral buckle, $360^{\circ}$ laser photocoagulation and postoperative silicon oil tamponade is effective $83.3 \%$ (20/24 eyes) in the management of GRT more than $180^{\circ} .{ }^{14}$ On the other hand, in a prospective randomized comparative study conducted by Sharma et al., they used $360^{\circ}$ degree $9 \mathrm{~mm}$ silicone band buckle in 10 cases and none in 11 cases. They reported that the primary success was $100 \%$ in sclera buckle group as compared to $37.5 \%$ in non sclera buckle group, and that re-operation were required in 8 out of 11 cases in non- scleral buckle group. The final visual acuity was better in eyes treated with scleral buckle.

Controversy remains whether lens extraction is necessary or not in the management of fresh giant tears. The advantage of lens removal is the better visualization of vitreous base. In this study, it was found that lens removal was not necessary and may minimize the surgical trauma in such complex procedure. In addition, intraocular lens power calculation is often inaccurate in eyes with GRT when the macula is off. Moreover, the use of wide angle viewing systems coupled with indentation for giant tear surgery improves the ability to see the peripheral retina in phakic and pseudophakic eyes and makes thorough vitreous base shaving feasible. ${ }^{1,3,9} \mathrm{In}$ this 
study, lensectomy performed on 4 eyes due to lens opacity. Sharma et al., considered lens removal only in many eyes with GRT are often highly myopic and the pars plana region is often wide and broad. This anatomic variation allows adequate exposure of the vitreous base with less risk of lens touch. ${ }^{15}$ However, the initial surgery for GRT should not be compromised to preserve the lens. In the current study, developed cataract in phakic eyes and lens extraction was done during silicon oil removal thus avoiding disadvantages of multiple surgeries.

Heavy liquids (perfluorocarbons) have revolutionized GRT surgery. They allow hydraulic manipulation of the retina. Their high specific gravity enables the retinal flap to be unrolled, and displacement of subretinal fluid in a posterior to anterior direction allows surgery to be performed with the patient in a supine position. Due to their high surface tension, retinal breaks can be closed internally, without the heavy liquid flowing into the subretinal space..$^{1-3}$ In this study, where at initial surgeries heavy liquid were used in 15/26 cases (57\%), 7 eyes had re-detachment (47\%). In the 11 eyes where heavy liquid was not used. The re-detachment rate was $64 \%(7 / 11)$. These results are in keeping with previous studies advocating the use of heavy liquid in the management of GRT.

Expansile gases and silicone oil have been used as tamponade in GRT surgery. Since the first report of the use of silicone oil for the treatment of retinal detachment in 1962, this agent has been increasingly used as a tamponade in managing complex retinal detachments including those resulting from GRT. The Silicon Oil Study has shown that, in eyes with complex retinal detachment, the retinal re-attachment rates, visual acuity outcomes, and complication rates are better with silicon oil compared to Sulphur hexafluoride gas as an internal tamponade, and comparable with C3F8. ${ }^{16}$ In this study, the use of silicone oil may have contributed to the anatomical success rate $50 \%$ (12/24 eyes), expansile gases has re-detachment rate was $100 \%$ (2/2). Garcia et al., said that superior GRT with retinal detachment and without PVR, prefer to use a mixture of SF6 gas. If there is PVR and tears at any location except for 5,6 , and 7 o'clock they use a mixture of C3F8. These patients should be able to position for at least 1 week. Finally, in detachments with severe PVR or tears in areas near 6 o'clock, they use 1000 centistoke SO. They do not support the frequent use of heavy SO tamponades, such as liquids and per fluorinated oils, because they provide inadequate protection against superior tears, are difficult to remove, and are toxic to the photoreceptors in the lower sectors of the retina. ${ }^{17}$

In this study, the success rate with primary procedure was $42 \%$ (11/26 eyes), which increased to $54 \%(14 / 26)$ with multiple surgeries. Visual acuity at the end of follow up period visual acuity improved in 11 (42\%) eyes. Our final retinal re-attachment rate was less than reported in cataractous eyes, subluxated lenses or the presence of PVR are the main indications for lens removal in GRT. Also, some of the other studies. This was due to recurrent retinal detachment in 15 cases, resulting from severe PVR (42\%). Shunmugam et al, reported primary and final retinal re-attachment rates are achieved in $88 \%$ and $95 \%$ of patients respectively. ${ }^{6}$ Even when the retina remains attached, however, visual recovery may be limited.

Prophylactic treatment of the fellow eye is controversial. Some authors suggest that fellow eyes of those with non-traumatic GRT should be treated prophylactically. ${ }^{18}$ In this study, prophylactic laser of the fellow eye in nontraumatic cases of GRT was 5/20 eyes (25\%).

\section{CONCLUSION}

In this study, the more common etiology GRT was high myopia, the majority gender was males. Mostly GRT size was $90^{\circ}$ with the most location was at temporal quadrant. The management were PPV alone and combined PPV with encircling scleral buckle. Anatomical success was $54 \%$, and visual acuity outcome in our case series were variable, the most was no change (54\%). This is a lack in our study, which should be measured is best corrected visual acuity.

\section{REFERENCES}

1. Beroccal $M H$, chenworth $M L$, Acaba LA: Management of Giant Retinal Detachments. J Ophthalmic Vis Res 2017, 12(1):93-97

2. Nagpal M. Giant retinal tears: Size does matter. Retina Today 2013, 26-28.

3. Adelman RA, Parnes AJ, Sipperley JO, Ducournau $D$ : Strategy for the Management of ComplexRetinal Detachments: The European Vitreo-Retinal Society Retinal Detachment Study Report 2. Ophthalmology 2013, 120:1809-1813

4. Mehdizadeh $M$, Afarid $M$, Hagigi MS. Risk factors for giant retinal tears. J Ophthalmic Vis Res 2010, 5:246-249.

5. Gonzalez MA, Flynn HW Jr, Smiddy WE, Albini TA, Tenzel P: Surgery for retinal detachment in patients with giant retinal tear: etiologies, management strategies and outcomes. Ophthalmic Surg Laser Imaging Retina 2013, 44(3):232-237.

6. Shunmugam M, Ang GS, Lois, N. Giant retinal tears. Surv Ophthalmol 2014, 59:192-216.

7. Ghosh YK, Banarjee S, Savant V, Kotamarthi V, Benson MT, Scott RAH and Tyagi AK. Surgical treatment and outcome of patients with giant retinal tears. Eye 2004, 18:996-1000

8. Chang S, Lopez JM. Giant retinal tears and proliferative vitreoretinopathy. In: Ryan SJ, ed. Retina. 4th ed. St. Louis: Mosby; 2006: 2345-2351.

9. Gopal L, Sharma T, Bhende PS. Complicated 
Forms of Retinal Detachment: Giant Retinal Tear. In: Ryan SJ, ed Retina. $5^{\text {th }}$ ed. St. Louis: Mosby; 2013: 1844-1851

10. Ang GS, Townend J, Lois N. Epidemiology of giant retinal tears in the United Kingdom: The British giant retinal tear epidemiology eye study (BGEES). Retina 2010, 51:4781-4787.

11. Gonzalez MA, Flynn H. Retinal Detachment Caused By Giant Retinal Tears: Etiologies, Tchniques and Outcomes. Investigate Ophthalmology \& Visual Science 2012, 53:58085810

12. Lee SY, Ong SG, Wong DWK, Ang CL. Giant retinal tear management: an Asian experience. Eye (Lond). 2009, 23(3):601-605

13. Ambresin A, Wolfensberger TJ, Bovey EH. Management of Giant retinal tears with vitrectomy, internal tamponade, and peripheral $360^{\circ}$ retinal photocoagulation. Retina 2003, 23(5):622
14. Dabour SA: The outcome of surgical management for giant retinal tear more than $180^{\circ}$. BMC Ophthlmology 2014, 14:86

15. Sharma $Y$, Reddy $P$, Azad R: 60 degree scleral buckling in vitreous surgery for giant retinal tears without proliferative vitreoretinopathy changes.Dulles, USA: American Academy of ophthalmology; 2000.

16. Vaziri K, Schwartz SG, Kishor KS, Flynn Jr HW. Tamponade in the surgical management of retinal detachment. Clinical Ophthalmology 2016, 10:471-476

17. Garcia RAG, Arencibia OD, Martinez FE. Surgery for Retinal Detachment with Giant Retinal Tears: An Update on Surgical Approaches. Retina Today 2014, 61-64

18. Kazahaya M. Prophylaxis of retinal detachment. Semin ophthalmol 1995, 10:79-86

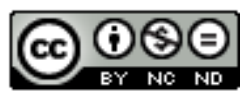

This work licensed under Creative Commons Attribution 